\title{
The Potential Applications of Machine Learning- Based Artificial Neural Networking Algorithms: A Review
}

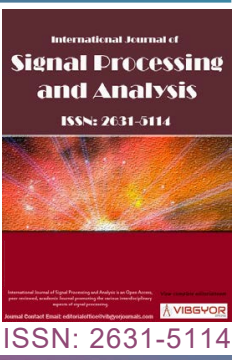

\section{Asma Channa ${ }^{1,2}$ and Muhammad Arif Mahmood ${ }^{3,4^{*}}$}

${ }^{1}$ Computer Science Department, University of Politehnica Bucharest, Romania

${ }^{2}$ DIIES Department, University Mediterranea of Reggio Calabria, Italy

${ }^{3}$ National Institute for Laser, Plasma and Radiation Physics (INFLPR), Romania

${ }^{4}$ Faculty of Physics, University of Bucharest, Romania

\begin{abstract}
Machine learning based artificial neural networking (ANN) has been acknowledged to be an authentic way to accomplish intricate pattern identification and regression analysis without any obvious necessity to paradigm and resolve the primary physical models. ANN has been introduced and adopted in various fields of life based-on their key advantages including learning and adapting ability, parallel distributed computation, robustness, and many more. This review articles discussesthe working principle, classes and structure of ANN. The potential applicable areas of ANN, the challenges and their solutions that have been identified through literature survey, are discussed and summarized.
\end{abstract}

\section{Keywords}

Machine learning, Artificial neural networking (ANN), Potential applications of ANN, Challenges and solutions in ANN

\section{Introduction}

During industrialization, remains a timeless manufacturing goal: To produce high quality products at minimum cost. In this context, manufacturing processes have intensely changed due to implementation of advanced manufacturing technologies specifically automation that can be done using computer programming and algorithms employing artificial intelligence (Al). In industrial $\mathrm{Al}$, the process known as "training", enables the ma- chine learning algorithms to detect anomalies correlations while performing test searching for patterns across the various data feeds, so facilitating the manufacturing process and making it easier for people to work [1].

Although, there are tremendous applications of $\mathrm{Al}$ in various aspects of manufacturing systems but expert systems are the most commonly used application of Al. Expert systems are commonly used in designing, scheduling, process planning,

*Corresponding author: Muhammad Arif Mahmood, National Institute for Laser, Plasma and Radiation Physics (INFLPR); Faculty of Physics, University of Bucharest, Magurele, Ilfov, 077125, Romania, Tel: +40214574491, Fax: +40-21-4574243; +40-21-4574467

Accepted: May 08, 2021; Published: May 10, 2021

Copyright: (c) 2021 Channa A, et al. This is an open-access article distributed under the terms of the Creative Commons Attribution License, which permits unrestricted use, distribution, and reproduction in any medium, provided the original author and source are credited.

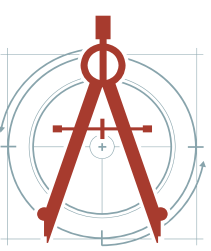

Channa et al. Int J Signal Process Anal 2021, 6:007 
quality control, and so on; However, there are various pros and cons as well such as Ali, et al. (1990) explained that the number of variables that must be engaged into consideration while neural model formation has become excessively abundant [2]. Due to vagueness and sheer extent of items to be considered in calculation approaches cannot rationally resolve the problem; Therefore, ample development needs to be done in both the neural network complexity and computing capacity prior to real-intelligent program formation [3]. These expert systems are found to be least efficient and effective in modern manufacturing systems due to complex, varying, and highly productive manufacturing environment.

In this modern era, there is a strong need of $\mathrm{Al}$ category that must capable to respond quick alternations within automated productive environment; furthermore, it must be able to learn and absorb quick changes, identify the trends, and forecast human being thoughts with minor human interferences. Moreover, it should identify the patterns and correlations among dominant factors which are not deceptive to human beings.

In the recent few years, several studies have been stated that artificial neural network (ANN) have proved their above mentioned-capabilities effectively in many practical areas such as planning, financial administration, investment and assurance amenities, engineering, and manufacturing systems [4-7]. ANN is one of the most interesting top- ics of research, and an incredible quantity of literature has been produced, in the last 15 to 20 years, specifically on ANN, their abilities and implementation in various fields of life. However, this literature is extensively dispersed in various disciplines and publications.

The intention of this study is to present an overview of ANNs and their applications that make it appropriate for various aspects of life. This review article is divided into major three sections. The $1^{\text {st }}$ section discusses the working principle, classes and structure of ANN. The potential applicable areas of ANN have been identified in $2^{\text {nd }}$ section. The $3^{\text {rd }}$ section discusses the challenges and their solutions that have been identified through literature survey. The purpose of this review is to present the readers an overall picture on concepts, applications and perspectives of ANN in various fields of life, thus providing some useful guidelines and references for the research and implementation.

\section{Artificial Neural Networking (ANN)}

In this section, introduction, working principle, various ANN classes, and structure of ANN.

\section{Introduction and working principle of ANN}

Machine learning (ML) can be classified into two major learnings: (a) Supervised ML and (b): Unsupervised ML. One of the easiest ways to identify between the two is to check whether the given dataset has labels on it or not. Therefore, ANN is identified as a type of supervised ML because in

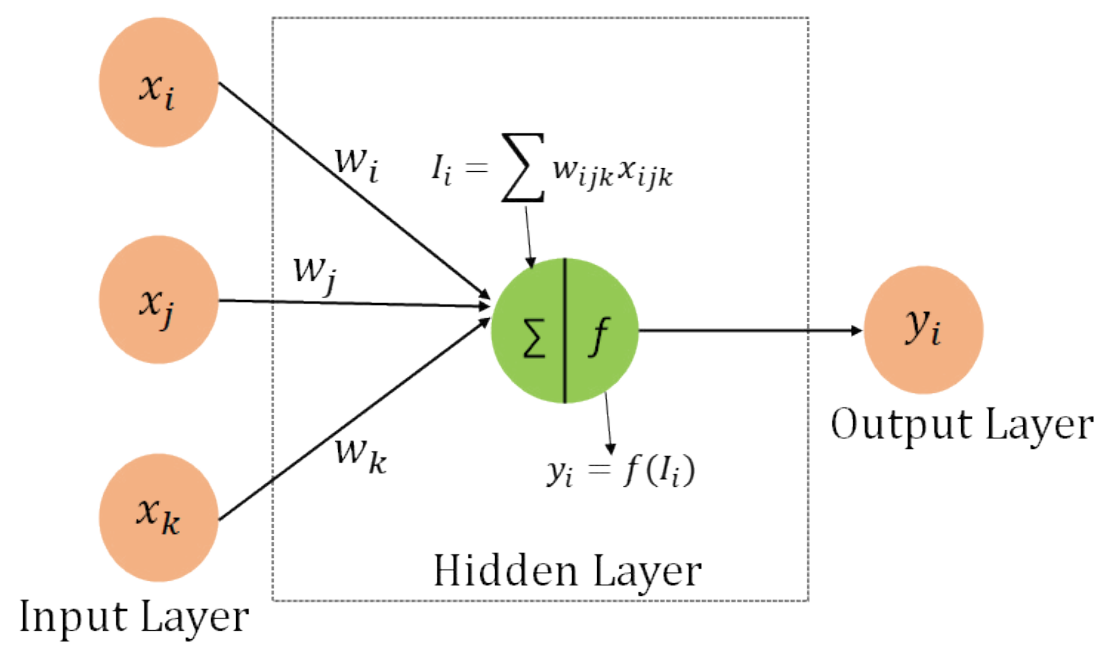

Figure 1: Feedforward data input schematic diagram. 
an ANN, the model has been told the response to the inputs. This makes suitable the ANN for various applications, since clear targets and requirement methods are already identified. An ANN has robust assessing techniques for complex and extremely nonlinear associations between the given input and output. It has been identified that a network having single hidden layer, but an adequate number of neurons can build an arbitrary function [8].

An ANN usually consist of three layers: (a) An input layer, (b) A hidden layer, and (c) An output layer [9], as shown in Figure 1 and Figure 2. Each layer contains several neurons or nodes, which is defined as the point where computation starts. The ANN calculates the connection magnitude of each input and assigns weightage of the given inputs. The weightage values are usually determined by training the ANN model iteratively, to minimize the loss function between the estimations and actual outputs. To train an ANN model, two methods are commonly used (a): Feedforward data input (FFDI) and (b): Backward Error Propagation (BEP) $[10,11]$. A comparison between the FID and BEP is given in the following Table 1 . The ANN has the capacity to predict the outputs for the unseen inputs, once the ANN model is trained.

\section{Various classes of ANN}

The three classes of ANN, given in Table 2, have proved their worthiness and gained popularity.

\section{Structure of ANN}

The structure of an ANN usually consists of four major sub-sections: (a) No. of hidden layers, (b) No. of neurons in each layer, (c) Activation function, (d) and loss estimation function. The details are summarized in Table 3.

\section{Applicable Areas for Artificial Neural Net- working (ANN)}

Figure 3 illustrates the possible various applications of ANN. It can be observed tremendous efforts have been carried out by a lot of researchers to implement ANN in their respective fields, which identifies the potential of ANN algorithms to reach for a feasible solution with the designed inputs.

\section{Resource management and allocation}

Hopfield and Tank (1982 and 1985) carried out numerous studies on the application of ANN in resource management and allocation, transport phenomena, and manufacturing systems [30,31]. They used ANN to find out the rapid solution of salesman problem (SP) and validate that ANN can be used to find out the quick results of a situation which is hard to analyze using traditional methods. An analog circuit along with the SP plotting was used to validate the computing power and speed of an analogous and immensely connected neurons networks. A map and graph K-coloring problem using Hopfield network was solved by Dahl (1987). Initially, a 100-region map problem (NP-Complete) was proved, and then transformed into SP before implementing an ANN to achieve an optimum solution [32]. Moreover, Hopfield network was used by Page and Tagliarini (1988) in order to resolve the

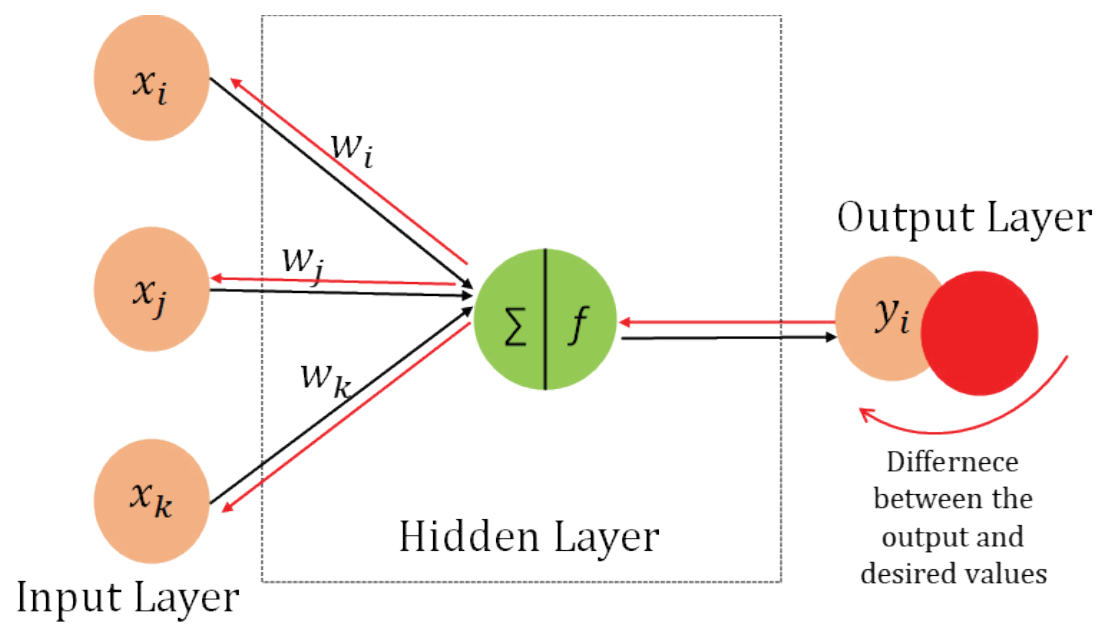

Figure 2: Backward Error Propagation Network schematic diagram. 
Table 1: Comparison between forward feedback data input and back error propagation [10-12].

\section{Feedforward data input (FFDI)}

- All the layers feed into one another from the input till output in the forward direction.

- By default, the architecture of FFDI assumes that all the nodes in a given layer are linked to those in the next layer.

- Therefore, the architecture of FFDI is almost fully defined except the loss function that is augmented in the output layer.

- The perceptron algorithm uses the perceptron criterion, this is not the only choice.

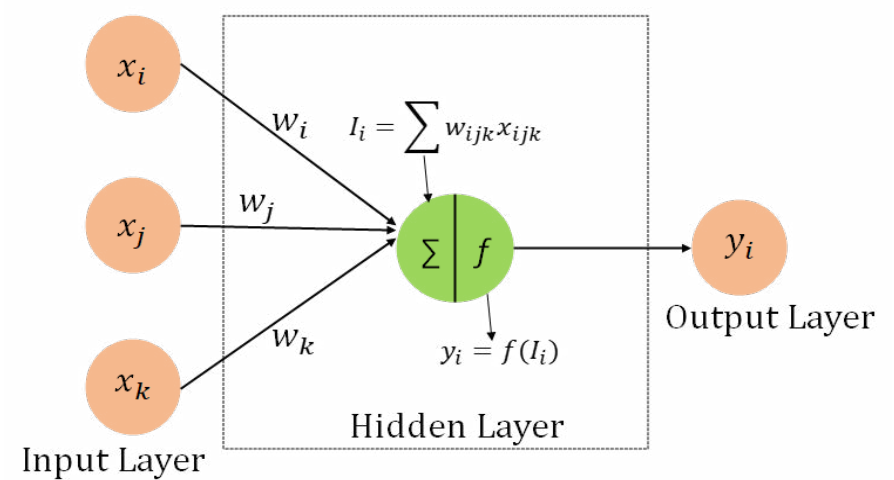

Figure 1. Feedforward data input schematic diagram.

\section{Backward Error Propagation (BEP)}

- It utilizes the chain rules defined mathematically to calculate gradients for each layer iteratively.

- It the most commonly used network for various applications.

- It is classified into two main phases: (a) Forward phase and (b) Backward phase.

o The forward phase is used to calculate the outputs and derivatives at different local nodes.

o The backward phase is used to collect the products of these local values all over the paths from the starting node till the output.

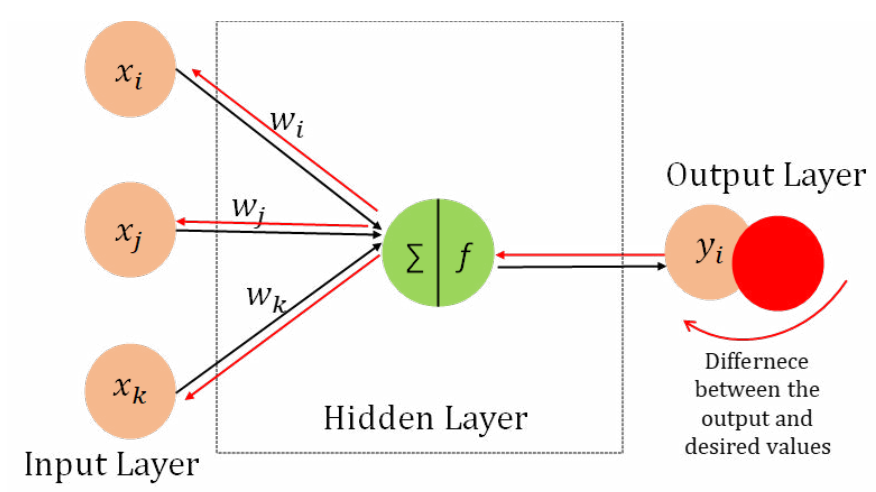

Figure 2: Backward Error Propagation Network schematic diagram.

Table 2: Three commonly used ANN models.

\section{Multilayer \\ Perceptron}

Convolutional Neural It deliberates the relationship between image pixels, therefore, used in image processing Network

Recurrent Neural Network

It is the most typical ANN; It is common in linear and nonlinear summations such as sigmoid function. It is commonly used for tabular data [13]. [14]. It plays a significant role in temporal dynamics, as it can build a connection between the number of nodes in a given layer. Therefore, it is used in long short-term memory that can accurately reproduces the simulations results [15]. 
Table 3: Structure of ANN model.

No. of hidden layers

No. of neurons in a given layer

Activation function

Loss estimation function
A combination of input-hidden-output layer with respective number of neurons is used to defined the ANN model $[16,17]$. For instance, 5-8-1 for a given ANN model means that input layer has 5 neurons, hidden layer has 8 neurons while, output layer has 1 neuron.

The required number of neurons in the input and output layer depends on the given problem. However, the number of neurons in the neurons must be chosen carefully. If the number of neurons are not selected optimally, it will lead to under- or over-fitting in the given dataset [18]. Various studies have been carried that suggested to choose the number of neurons in between 5 10 [19-28].

It is a non-linear transformation on given the input signal. In other words, it makes a decision to activate and deactivate a given neuron. On the basis of its performance, it is considered a vital part of an ANN model. It is important to mention that a network without having an activation function behaves like a model for linear regression, thus, cannot handle the complicated tasks [29]. Some commonly used activation functions are:

$$
\begin{aligned}
& \operatorname{ReLU}(\mathrm{x})=\max (0, x)(1) \\
& \operatorname{Tanh}(x)=\frac{2}{1-e^{-2 x}}-1(2) \\
& \operatorname{Sigmoid}(x)=\frac{1}{1-e^{-x}}(3)
\end{aligned}
$$

The loss function is usually determined using the real-world problem, carries the interpretation real-time data. The root means square (RMS) and absolute mean error (AME) are two commonly used ways to estimate the difference between the predicted vector and the target value [8]. Their mathematical expressions are given below:

$$
\begin{aligned}
& R M S=\sqrt{\frac{\sum_{i=1}^{n}\left(x_{i}-x_{t}\right)^{2}}{n}} \text { (4) } \\
& A M E=\frac{\sum_{i=1}^{n}\left|x_{i}-x_{t}\right|}{n} \text { (5) }
\end{aligned}
$$

where $\mathrm{i}$ a sample index, $\mathrm{x}_{\mathrm{i}}$ is a predicted value and $\mathrm{x}_{\mathrm{t}}$ is the target value.

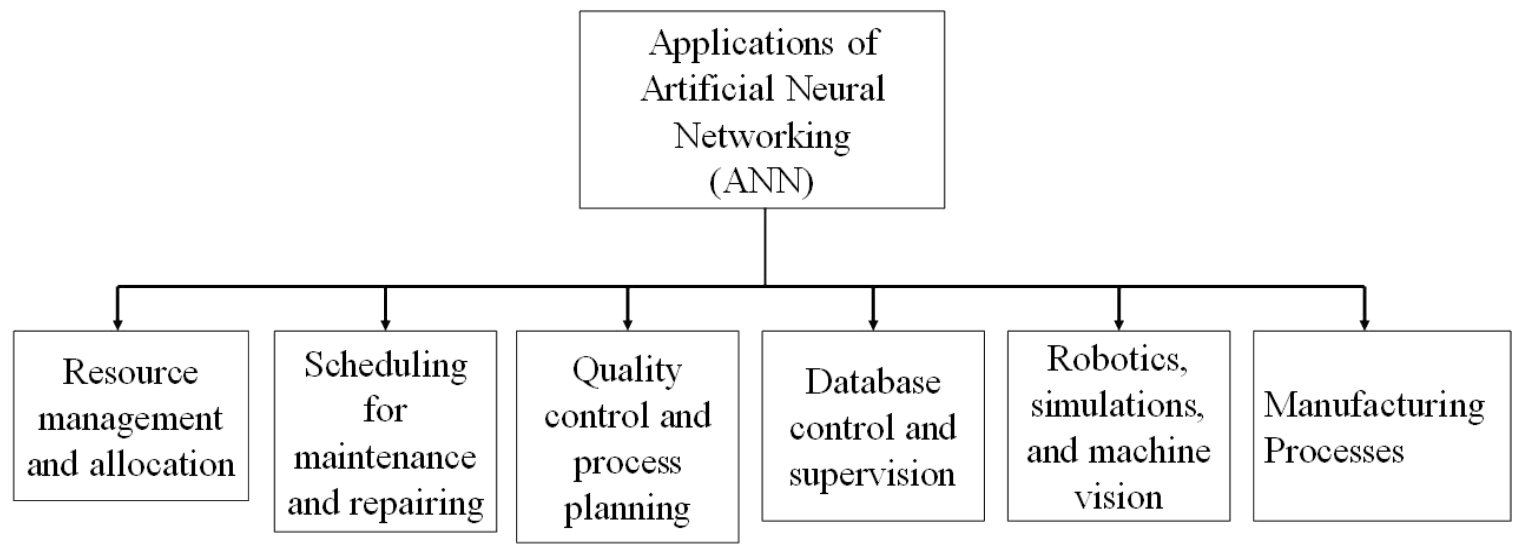

Figure 3: Classification of artificial neural networking applications in various fields.

problem related to resource allocation [33]. Tangliarini and Page (1987) implemented ANN technique to resolve queens' problems; the results show that ANN resulted in ninety (90) achievable solutions out of ninety-two problem constraints [34]. 
Marcus (1987) designed an approach, with a set of algorithms, alternative to the Hopfield's networking strategy; the results show that Marcus' algorithm is proficient in producing solutions analogous to Hopfield' solutions [35]. Levy and Adams (1987) presented an optimization strategy which combines computational annealing procedure with Hopfield' network, found to be much more efficient than Hopfield' strategy [36]. It has been that ANN reduces the computation time as compared to the conventional problem-solving methods. Saylor and Stork (1986) modelled a decision-making procedure using ANN, found to be effective and consumes less time in comparison to a conventional technique [37]. Moreover, Udo (1991) solved warehousing problems using ANN, and the results indicated that the ANN algorithms performed efficiently with the reduction in $66 \%$ computation time and average memory storage [38].

\section{Scheduling for maintenance and repairing}

Various researches have been carried to implement ANN in maintenance and repairing planning and solutions. In this scenario, Chaudhri and De (2010) developed Rough Fuzzy Multi-Layer Perception ANN for job scheduling problems. ANN algorithms produce optimal schedules for the given inputs, which enhances the organization strength, and apprehends the predictive knowledge linked to the assignment of task to operators in sequence [39]. Foo (1998) used an ANN algorithm to resolve 4-men with 3-machines (men-machine) scheduling problem that resulted in an optimum solution with the given inputs [40]. In addition, Foo and Takefuji (1987) developed an ANN with linear programming (ANNLP) and used to solve man-machine job problem; the results exhibited a near to optimal solution can be obtained using ANNLP [41]. Rabelo and Alptekin (1989) proposed an algorithm, flexible manufacturing systems (FMS), in which ANN in combination with expert manufacturing systems were used to produce optimum solutions. The designed algorithms resulted in quality decision making that leads to quality enhancement in manufacturing processes [42]. Noaker (1988) proposed that the technology must be employed for six functions mainly: (1) Locating, (2) Condition analysis, (3) Absence recognition, (4) Examination, (5) Identifica- tion of condition, and (6) With the emphasis on detection when the conditions are no more favorable and fixing them using proactive approach [43].

Subbaraj and Kannapiran (2010) presented the design and development of an ANN model for the fault detection of Pneumatic valve in cooling system with the capability to identify total nineteen faults. The model was trained using real time data, and the predicted results were found close to the real-time results [44]. Ray (1991) applied ANN in engine fault analysis; the results obtained from the case study explained that ANN provided the analogues judgements as human experts with the efficiency of $75 \%$ time [45]. Yam, et al. (2001) reported a study on implementation of ANN on condition-based maintenance. In their study, an intelligent predictive decision system model, using the recurrent artificial neural network method, was established and verified for equipment in a power plant; the results presented that the ANN model provided the fault diagnosis with an accuracy, and predicted the equipment life while considering the deterioration [46]. Oladokun, et al. (2006) implemented the ANN for maintenance management; the results show that the model can predict the expected downtime with $70 \%$ accuracy [47].

\section{Quality control and process planning}

Various researches have been carried on the implementation of ANN for quality control and process planning. In this regard, Chang and Jiang (2002) developed an ANN model to review the dependency between the quality of finished product and sensor measurements to examine the tool failure during manufacturing [48]. Port (1990) reported on the implementation of ANN in "Anderson Company" w.r.t quality inspection with the intention to improve the performance from 350 per million-100 per million [47].

Thomas (1995) explained that ANNs can be used to control a process statistically, classify the kind of variations had occurred within the system [49]. In order to identify the defects in loudspeakers, an ANN strategy was implemented by "CTS electronics" that resulted in precise, faster, and accurate inspection. Moreover, Ford motor implemented the ANN models to inspect the paint quality of cars 
and to detect the engine problems [50]. English and Sastri (1990) explained a new technique regarding quality control in a steady-state manufacturing process using ANN technique and a close correlation was found between ANN model prediction results and real life results [51]. Patrick (1991) presented an application of ANN on washing operations. The results indicated a $25 \%$ improvement washing efficiency with good quality, and improved profits [52].

\section{Database control and supervision}

ANN can be used to control, manage, and supervise database. Bandara, et al. (2020) presented an estimation model that can be used with various recurrent neural network models. The model was tested using long short-term memory networks. A closed correlation was found between competitive outcomes and benchmarked datasets [53]. Amorim, et al. (2019) implemented semi-supervised techniques to categorize unlabeled trainings set with the training on convolution ANN. The technique was experimentally tested to identify soybean leaf, and herbivorous pests [54]. Janowski and Horzyk (2018) presented an ANN model for maze examination and knowledge-based adaptation. Their unique approach is beneficial for the situations when there is no initially well-defined set of data for training, but can be developed gradually within training progression based on the factors that can be introduced from the working environment [55].

Shahid, et al. (2019) reported that health care organizations are using ANN technique to identify health care management decisions from a given database [56]. Hillman (1990) presented a technique in which ANN and expert systems are collectively used to develop a database; the results exhibited that an increment in productivity was found without a declination in the final product quality [57]. An application of ANN in database management field was reported by Lelu (1991); Test or multimedia-based browser can be placed and queried using this database management technique [58].

\section{Robotics, simulations and machine vision}

Wang, et al. (2019) presented a unique technique using robot learning demonstration. In this technique, an ANN-based controller was designed for the robot to track and identify the trajectories created using motion-model, ANN radial function was used to accumulate the consequences generated using dynamic situations. The experimental results performed using a robot (Baxter), were validated using ANN model [59]. Chen, et al. (2019), proposed a technique with constrained optimization recurrent neural network so that mobile robots can be handled easily; a comparison was made between numerical simulations and existing literature on recurrent neural network which resulted in good understanding between the two [60]. Zheng, et al. (2019) presented a robust design for controller to control the movement of a robot. An ANN model was used to estimate the relation between the actuators and the position of the robot (soft); The reliable results can be achieved using Zheng' robust controller [61].

ANN are commonly used in simulations as well. Sahiner and Liu (2020) proposed an improved method for the analyses of gamma spectra produced by neutron, using an ANN model in combination with Monte Carlo simulations. The model can predict the concentrations of elements within a range of absolute errors (1.8 2.8\%) [62].

\section{Manufacturing operations}

Efficiency, quality, and production in a manufacturing system can be enhanced using computer assisted processing and planning by reducing lead times and expenses by implementing improved manufacturing practices thus improving competitiveness in the market. CAPP attempts to capture the thoughts and methods of the experienced process planner. Variant systems are understandable, generative systems can plan new parts. Expert systems increase flexibility, fuzzy logic captures vague knowledge while neural networks learn. The combination of fuzzy, neural and expert system technologies is necessary to capture and utilize the process planning logic. A system that maintains the dependability and clarity of variant systems, is capable of planning new parts, and improves itself through learning is needed by industry.

Various researches have been carried out in injection molding (IM) processes. Bensingh, et al. (2019) implemented hybrid ANN model and parti- 
cle swarm optimization (PSO) technique in IM process of lens (bi-aspheric) to achieve least variation in volume shrinkage, which is more challenging and crucial for the optical quality. The developed hybrid network was trained and tested using experimental for the optimization of the IM process parameters. It was observed that the developed hybrid ANN model presented the optimized IM process parameters of the bi-aspheric lens having an optical power equal to 27.73 Diopter and seventh order spherical abnormalities [63]. Everett and Dubay (2017) designed and developed an ANN model to regulate the process parameters to govern the mold cavity temperature in IM process. The results demonstrated that the developed ANN model accurately predicted the dynamic performance of the mold cavity temperature. This is one of the keys in many industrial applications, where the critical factors in a system are not directly observable [64]. Yin, et al. (2011) developed a Back-Propagation ANN model for warpage estimation and optimization of IM parts based on the operating process parameters. The finite Element (FE) simulations were carried out on the basis of input and output, and the results of FE simulations were used to train the developed the ANN model. It was found that the ANN model has the ability to estimate the warpage in IM parts within a range of $2 \%$ error. Moreover, ANN has the capability to optimize process parameters including energy consumption and production cycle. The optimized warpage value equal to $1.58 \mathrm{~mm}$ was achieved with ANN model, which is $32.99 \%$ shorter than the initial warpage $(2.358 \mathrm{~mm})$, and the cooling time was reduced from 20 to 10 s [65].

The ANN models are widely used by various researchers in the forming processes. Monajati, et al. (2010) used a feed forward back-propagation neural network model to illustrate the influence of operating parameters on the evolution of mechanical properties and formability of steel sheets deep drawing. This model contains nineteen operating parameters. After the training process of ANN model, the results showed that the model can be used in a quantitative way to guide and control the final properties of formed low carbon steel items [66]. Downes and Hartley (2006) developed an ANN model to assist the design of roll in cold-roll forming processes, which can deliver guidance to the inexpert designers to design a better roll on the basis of input parameters [67]. Brahme, et al. (2009) presented an ANN model-based model for the prediction of textures generated in cold rolling of steels sheets. A feedforward interconnected ANN with standard backpropagation error algorithm was used. The texture data in the form of texture intensities including amount of carbon, carbide magnitude and the amount of reduction ratio was used as input to train the model while the output of the model was in the form of fiber texture. It was found that the predictions of the ANN can provide an excellent match between experimental data and estimated analyses [68].

In addition, ANN model has been implemented in the electric discharge machining (EDM) process. Surya, et al. (2017) developed an ANN model to predict the machining parameters in case of Wire-EDM of $\mathrm{Al} 7075+\mathrm{TiB}_{2}$ composites to attain maximum material removal rate, minimum dimensional errors and better surface roughness. The controlling factors indulged during the study were pulse-on and-off time, and current and bed speeds. The predicted parameters were found in the good agreement with the experimental ones within a range of $0.003 \sim 3.87 \%$ for material removal rate, $1.3 \sim 12.51 \%$ for surface roughness and $0.03 \sim 1.31 \%$ for dimensional accuracy [69]. Ugrasen, et al. (2014) presented a study on optimization and influence of machining parameters on precision, roughness and volumetric material removal rate of manufactured parts in Wire-EDM process. The important machining parameters influencing the precision, roughness and volumetric material removal rate were identified using analyses of variance method. The controlling factors identified were Pulse-on and-off time, Current and Bed speed. An ANN model with back propagation feed forward neural network and Levenberg-Marquardt algorithm was used to establish and train the model. It was found that the $70 \%$ training data set correlated well with the experimental parameters [70]. Vishnu, et al. (2018) established an analytical model in combination with an ANN model (back propagation algorithm) for machining Inconel-718 via EDM using various cutting conditions of polarity, pulse on- and -off 
time, and peak most current. The material removal rate, surface roughness and cutting tool wear rate were selected as output. Sufficient level of fitness was observed for the trained model. A comparison was made between experimental and the estimated analyses, which shows that ANN can estimate the values with an accuracy of $98.82 \%, 88.02 \%$, and 93.97\% for material removal rate, cutting tool wear rate, and surface roughness, respectively [71].

Various applications can be found for the implementation of ANN in casting process. Soundararajan, et al. (2017) implemented an ANN model (feed forward back) and full factorial design to measure the influence of operating variables on mechanical properties of $A 413+B_{4} C$ composites produced by squeeze casting. The designed ANN was found in the good agreement with the experimental data having mean-correlation and percentage error equal to 0.95 and 0.87 , respectively [72]. Tirian, et al. (2014) presented an ANN-based technique to predict crack formation designed to improve the casting process of steel. The output of the ANN was used as an input in fuzzy decision system considered as decision block. The proposed system was found capable enough to estimate crack formation for the given operating conditions, moreover, it can be implemented in any system with minimum adaption [73]. Yu, et al. (2009) developed an ANN (three-layer back propagation) to predict the performance of porous $\mathrm{Si}_{3} \mathrm{~N}_{4}$ ceramics. In $\mathrm{ANN}$, the effects of compositions on the performance of porous $\mathrm{Si}_{3} \mathrm{~N}_{4}$ ceramics were taken into account. By using the ANN model, it was found that the approach presented the encouraging predictions for the performances of porous $\mathrm{Si}_{3} \mathrm{~N}_{4}$ ceramics, which shows that the ANN can be used as a very useful tool to analyze the performance of $\mathrm{Si}_{3} \mathrm{~N}_{4}$ [74].

The ANN tool has been extended to additive manufacturing applications. Yadav, et al. (2020) implemented an ANN model for the multi-material (50\% Acrylonitrile Butadiene Styrene $+50 \%$ Polyethylene Terephthalate Glycol) 3D printing by fused deposition modeling (FDM). The density and extrusion temperature were optimized to improve the tensile strength of FDM-ed parts. The results show that ANN has the capability to maximized tensile strength up to $4.54 \%$ [75]. Hu, et al. (2019) em- ployed ANN in stereolithography (SLA) technique. Three main operating parameters in SLA including orientation $\left(0^{\sim 9} 90^{\circ}\right)$, post curing time (20 60 min), and annealing time ( $0 \sim 4 \mathrm{hrs}$.) were used as input to build and train the ANN model with the emphasis on hardness as output. This study proves that the hardness of the SLA-ed objects can be optimized up to $1 \%$ error using ANN method [76].

\section{Potential Challenges in ANN and their Solu- tions}

Following potential challenges have been identified on the basis of literature survey.

\section{Data sets optimization}

The performance of an ANN model depends on the quantity and type of the data provided while training. Therefore, an ANN is a data-driven model. On the other hand, it is expensive and time consuming to collect and organize the data for the training of an ANN model. There are certain methods available that can enlarge a dataset in an artificial and one of the ways to do it is to use artificial generative models [77]. Kingma and Welling (2014) explained that the autoencoder has a capability to generate randomly new data sets, by keeping view the training data, the extension of this autoencoder is known as variational auto-encoder [78]. Other generative models, such as generative adversarial nets and adversarial autoencoders, can also provide ways to perform data augmentation $[79,80]$.

\section{Selection of significant input parameters}

The training of an ANN model majorly depends on the input parameters, while the operating parameters play a significant role in any process. A selection of excess number of input parameters can easily overfit the ANN model. Therefore, it is of great importance to confirm that the ANN model is trained and operating at optimum number of input parameters. There are two techniques available, given in Table 4, for pre-processing of given to find out the significant input parameters.

\section{Under- and over-fitting in ANN model}

The main objective of an ANN model is a good estimation of output on the basis of previously given input data. But this performance can be disturbed due to the over- or under-fitting of an ANN 
Table 4: Techniques for pre-processing of given data $[8,81]$.

\section{Feature selection}

This technique assists in determining the most influencing parameters from the given list of parameters using statistical tools.

\section{Illustration}

For instance, in order to find out which parameter is affecting significantly on the 3D printed part e.g., hatch distance, or laser power, or layer thickness. In this situation, a Pearson's coefficient will be determined between two parameters that will figure out the dependency between two parameters. If the Pearson's value (max. $=1$ ) is higher for one parameter as compared to the other parameter, it means it will be affecting my desired output significantly.

\section{Feature combination}

This technique helps to carry out dimensionality lessening for the input attributes, and thereby concentrating on the newly generated features. Once the translation regulation is identified, manual manipulations are usually preferred. Mathematical tools such as principle components analysis can be utilized for the same purpose on the basis of attribute.

\section{Illustration}

For example, energy density (ED) influences the solidification, metallurgical, microstructure, and mechanical properties of a 3D printed part. Laser power, scanning speed, hatch distance, and layer thickness combines and generates a new feature ED. The aforementioned four features control the ED. model on the basis of given input data. An overfit ANN algorithm means a model tries to fit itself on every data point within the given dataset, the model becomes vulnerable to noises within the dataset. In contrary, an underfit model means that the model has failed to dig-out the mandatory relationships among the data points in the training dataset. These problems can be avoided by selecting the optimum number of neurons within each layer [82,83].

\section{Linking the analytical modelling and numerical with ANN}

In order to avoid lots of experimentations and producing a pool of datasets for the optimization of ANN model, is to develop analytical or numerical simulations. The results of analytical simulations can be used to train the ANN models. Koeppe, et al. (2018) proposed a methodology that combined the experiments, Finite Element (FE) simulations and ANN. Initially, the experimental analyses the FE-simulations were conducted to validate FE-simulations. Followed on, 85 simulational analyses were carried out using various parametric combinations such as load, displacement, strut radius and cell scale (input), while the maximum Von-Mises and principal stresses were categorized as outputs. After training conducted for ANN model, a close correlation for the loading history was found between an ANN estimations and FEM simulations [84].

\section{Conclusion and Future Outlook}

This paper provides a comprehensive overview of the applications of ANN in various fields of life. Various researches have been carried, which proved the robustness and trustworthiness of ANN as potential candidate to estimate output on the basis of given inputs. The scope of this work covers many variants of ANNs in different scenarios including: Resource management and allocation, scheduling for maintenance and repairing, quality control and process planning, database control and supervision, robotics, simulations and machine vision, and manufacturing operations. However, the behavior of output strongly depends on the data provided to ANN for its training (input) and the number of neurons required in each layer (input-hidden-output). On the basis of the above fact, the problems encountered in ANN and their solutions are provided in current study. However, there are still more studies and investigations to be done to improve the application of ANN and to achieve the numerous benefits.

\section{References}

1. (2020) What is decision making ? Management Study Guide.

2. Hertz JA, Krogh AS, Palmer RG (1991) Introduction to 
the theory of neural computation. Westview Press, 44.

3. Ali AL, Ali DL, Ali KS (1990) The undeterministic manipulation of solid models for robot program synthesis. Comput Ind Eng 19: 465-468.

4. Chryssolouris G, Lee M, Domroese M (1991) The use of neural networks in determining operational policies for manufacturing systems. J Manuf Syst 10: 166-175.

5. Franke C (2017) Autonomous driving with a simulation trained convolutional neural network.

6. Navarro A, Joerdening J, Khalil R, Brown A, Asher Z (2018) Development of an autonomous vehicle control strategy using a single camera and deep neural networks. SAE Tech Pap SAE International.

7. Tweedie FJ, Singh S, Holmes DI (1996) Neural network applications in stylometry: The federalist papers. Lang Resour Eval 30: 1-10.

8. Qi X, Chen G, Li Y, Cheng X, Li C (2019) Applying neural-network-based machine learning to additive manufacturing: Current applications, challenges, and future perspectives. Engineering 5: 721-729.

9. Goldberg Y (2017) Neural network methods for natural language processing. Synth Lect Hum Lang Technol 10: 1-309.

10.(2020) Artificial neural network.

11.(2020) A beginner's guide to neural networks and deep learning. Pathmind.

12.Aggarwal CC (2018) Neural networks and deep learning.

13.Gardner MW, Dorling SR (1998) Artificial neural networks (the multilayer perceptron)-A review of applications in the atmospheric sciences. Atmos Environ 32: 2627-2636.

14. Krizhevsky A, Sutskever I, Hinton GE (2012) Imagenet classification with deep convolutional neural networks. Adv Neural Inf Process Syst 25: 1097-1105.

15. Mikolov T, Karafiát M, Burget L, Černocký J, Khudanpur S (2010) Recurrent neural network based language model. 11th Annu Conf Int Speech Commun Assoc ISCA Archive, 1045-1048.

16.Sood AK, Ohdar RK, Mahapatra SS (2012) Experimental investigation and empirical modelling of FDM process for compressive strength improvement. J Adv Res 3: 81-90.

17.Sood AK, Equbal A, Toppo V, Ohdar RK, Mahapatra SS (2012) An investigation on sliding wear of FDM built parts. CIRP J Manuf Sci Technol 5: 48-54.
18.Xu S, Chen L (2008) A novel approach for determining the optimal number of hidden layer neurons for FNN's and its application in data mining.

19.Li XF, Dong JH, Zhang YZ (2009) Modeling and applying of RBF neural network based on fuzzy clustering and pseudo-inverse method. Proc Int Conf Inf Eng Comput Sci ICIECS 2009.

20.Munguía J, Ciurana J, Riba C (2009) Neural-network-based model for build-time estimation in selective laser sintering. Proc Inst Mech Eng Part B J Eng Manuf 223: 995-1003.

21. Equbal A, Sood AK, Ohdar RK, Mahapatra SS (2011) Prediction of dimensional accuracy in fused deposition modelling: A fuzzy logic approach. Int J Product Qual Manag 7: 22-43.

22.Vosniakos GC, Maroulis T, Pantelis D (2007) A method for optimizing process parameters in layer-based rapid prototyping. Proc Inst Mech Eng Part B J Eng Manuf 221: 1329-1340.

23.Sood AK, Ohdar RK, Mahapatra SS (2010) Parametric appraisal of fused deposition modelling process using the grey Taguchi method. Proc Inst Mech Eng Part B J Eng Manuf 224: 135-145.

24.Chen H, Zhao YF (2015) Learning algorithm based modeling and process parameters recommendation system for Binder Jetting additive manufacturing process. Proc ASME Des Eng Tech Conf American Society of Mechanical Engineers (ASME).

25.Shen $X$, Yao J, Wang Y, Yang J (2004) Density prediction of selective laser sintering parts based on artificial neural network. International Symposium on Neural Networks 3174: 832-840.

26. Rong-Ji W, Xin-Hua L, Qing-Ding W, Lingling W (2009) Optimizing process parameters for selective laser sintering based on neural network and genetic algorithm. Int J Adv Manuf Technol 42: 1035-1042.

27.Garg A, Tai K, Savalani MM (2014) State-of-the-art in empirical modelling of rapid prototyping processes. Rapid Prototyp J 20: 164-178.

28. Wang CY, Jiang N, Chen ZL, Chen Y, Dong Q (2015) Prediction of sintering strength for selective laser sintering of polystyrene using artificial neural network. J Donghua Univ 20: 164-178.

29. Ioffe S, Szegedy C (2015) Batch normalization: Accelerating deep network training by reducing internal covariate shift. In: 32nd Int Conf Mach Learn ICML 2015, International Machine Learning Society (IMLS) 448-456.

30. Hopfield JJ, Tank DW (1985) Neural computation of 
decisions in optimization problems. Biological Cybernetics 52: 141-152.

31. Hopfield JJ (1982) Neural networks and physical systems with emergent collective computational abilities. Proc Natl Acad Sci USA 79: 2554-2558.

32.Dahl E (1987) Neural network algorithms for an np-complete problem: Map and graph coloring. IEEE 1st Int Conf Neural Networks, New York, 113-120.

33.Page EW, Tagliarini GA (1988) Algorithm development for neural networks. DP Casasent (Ed) 11.

34.Tagliarini GA, Page EW (1987) Solving constraint satisfaction problems with neural networks. IEEE 1st Int Conf Neural Networks, San Diego, USA, 741-747.

35. Marcus R (1987) A connectionist algorithm for minimum cost network flows. IEEE 1st Int Conf Neural Networks, San Diego, USA, 735-739.

36.Levy BC, Adams MB (1987) Global optimization with stochastic neural networks. IEEE 1st Int Conf Neural Networks, San Diego, USA, 681-389.

37. Saylor J, Stork DG (2008) Parallel analog neural networks for tree searching. AIP Publishing 151: 392397.

38.Udo GJ (1991) Neural network applications to combinatorial optimization problems. Process Decis Sci Inst 491-493.

39.Chaudhuri A, De K (2010) Job scheduling problem using rough fuzzy multilayer perception neural networks.

40.Foo YS (1988) Neural network optimization techniques for job-shop scheduling. Dept of Electrical and Computer Engineering, University of South Carolina USA.

41.Foo YS, Takefuji Y (1987) Integer linear programming neural networks for job-shop schedulings. IEEE 1st Int Conf Neural Networks, 341-348.

42.Rabelo LC, Alptekin S (1989) Integrating scheduling and control functions in computer integrated manufacturing using artificial intelligence. Comput Ind Eng 17: 101-106.

43. Noaker PM (1988) Smart sensing on the factory floor. Production 100: 11-19.

44.Subbaraj P, Sangam K (2010) Artificial neural network approach for fault detection in pneumatic valve in cooler water spray system. International Journal of Computer Applications.

45.Ray AK (1991) Equipment fault diagnosis-A neural network approach. Comput Ind 16: 169-177.
46.Yam RCM, Tse PW, Li L, Tu P (2001) Intelligent predictive decision support system for condition-based maintenance. Int J Adv Manuf Technol 17: 383-391.

47. Oladokun VO, Charles-Owaba OE, Nwaouzru CS (2006) An application of artificial neural network to maintenance management. J Ind Eng Int 2: 19-26.

48.Chang DS, Jiang ST (2002) Assessing quality performance based on the on-line sensor measurements using neural networks. Comput Ind Eng 42: 417-424.

49.Stützle T (1955) A neural network approach to quality control charts. In: Lect Notes Comput Sci (Including Subser Lect Notes Artif Intell Lect Notes Bioinformatics), Springer Verlag, 1135-1141.

50. Kirrane DE (1990) Machine learning. Train Dev 40: 24-29.

51.English JR, Sastri T (1990) Enhanced quality control in continuous flow processes. Comput Ind Eng 19: 258-262.

52.Patrick KL (1991) Neural network keeps BSW filtrate solide at maximum uniform levels. Pulp Pap 65: 5558.

53.Bandara K, Bergmeir C, Smyl S (2020) Forecasting across time series databases using recurrent neural networks on groups of similar series: A clustering approach. Expert Syst Appl 140: 112896.

54.Amorim WP, Tetila EC, Pistori H, Papa JP (2019) Semi-supervised learning with convolutional neural networks for UAV images automatic recognition. Comput Electron Agric 164: 104932.

55.Janowski M, Horzyk A (2018) Supervised neural network learning with an environment adapted supervision based on motivation learning factors. Lect Notes Comput Sci (Including Subser Lect Notes Artif Intell Lect Notes Bioinformatics), Springer Verlag, 76-87.

56. Shahid N, Rappon T, Berta W (2019) Applications of artificial neural networks in health care organizational decision-making: A scoping review. PLoS One 14: e0212356.

57.Hillman DV (1990) Integrating neural networks and expert systems. Al Expert 5: 54-58.

58. Lelu A (1991) From data analysis to neural networks: new prospects for efficient browsing through databases. J Inf Sci 17: 1-12.

59. Wang N, Chen C, Yang C (2019) A robot learning framework based on adaptive admittance control and generalizable motion modeling with neural network controller. Neurocomputing 390: 260-267.

60.Chen D, Li S, Liao L (2019) A recurrent neural net- 
work applied to optimal motion control of mobile robots with physical constraints. Appl Soft Comput J 85: 105880.

61.Zheng G, Zhou Y, Ju M (2019) Robust control of a silicone soft robot using neural networks. ISA Trans 100: 38-45.

62.Sahiner H, Liu X (2019) Gamma spectral analysis by artificial neural network coupled with monte carlo simulations. Nucl Instruments Methods Phys Res Sect A: Accel Spectrometers Detect Assoc Equip 953: 163062.

63.Bensingh RJ, Machavaram R, Boopathy SR, Jebaraj C (2019) Injection molding process optimization of a bi-aspheric lens using hybrid artificial neural networks (ANNs) and particle swarm optimization (PSO). Meas J Int Meas Confed 134: 359-374.

64.Everett SE, Dubay R (2017) A sub-space artificial neural network for mold cooling in injection molding. Expert Syst Appl 79: 358-371.

65.Yin F, Mao H, Hua L, Guo W, Shu M (2011) Back Propagation neural network modeling for warpage prediction and optimization of plastic products during injection molding. Mater Des 32: 1844-1850.

66. Monajati H, Asefi D, Parsapour A, Abbasi S (2010) Analysis of the effects of processing parameters on mechanical properties and formability of cold rolled low carbon steel sheets using neural networks. Comput Mater Sci 49: 876-881.

67.Downes A, Hartley P (2006) Using an artificial neural network to assist roll design in cold roll-forming processes. J Mater Process Technol 177: 319-322.

68.Brahme A, Winning M, Raabe D (2009) Prediction of cold rolling texture of steels using an artificial neural network. Comput Mater Sci 46: 800-804.

69.Surya VR, Kumar KMV, Keshavamurthy R, Ugrasen G, Ravindra HV (2017) Prediction of machining characteristics using artificial neural network in wire EDM of al7075 based in-situ composite. Mater Today Proc Elsevier Ltd 4: 203-212.

70.Ugrasen G, Ravindra HV, Prakash GVN, Keshavamurthy $R$ (2014) Process optimization and estimation of machining performances using artificial neural network in wire EDM. Procedia Mater Sci 6: 1752-1760.

71.Vishnu P, Santhosh Kumar N, Manohar M (2018) Performance prediction of electric discharge machining of inconel-718 using artificial neural network. Mater Today Proc Elsevier Ltd 5: 3770-3780.

72.Soundararajan R, Ramesh A, Sivasankaran S, Vignesh M (2017) Modeling and analysis of mechanical prop- erties of aluminium alloy (a413) reinforced with boron carbide (b4c) processed through squeeze casting process using artificial neural network model and statistical technique. Mater Today Proc Elsevier Ltd 4: 2008-2030.

73. Tirian GO, Filip I, Proştean G (2014) Adaptive control system for continuous steel casting based on neural networks and fuzzy logic. Neurocomputing 125: 236245.

74. Yu J, Wang H, Zhang J (2009) Neural network modeling and analysis of gel casting preparation of porous Si3N4 ceramics. Ceram Int 35: 2943-2950.

75.Yadav D, Chhabra D, Kumar Garg R, Ahlawat A, Phogat A (2019) Optimization of FDM 3D printing process parameters for multi-material using artificial neural network. Mater Today Proc 21: 1583-1591.

76. Hu G, Cao Z, Hopkins M, Hayes C, Daly M, et al. (2019) Optimizing the hardness of SLA printed objects by using the neural network and genetic algorithm. Procedia Manuf 38: 117-124.

77. Géron A (2019) Hands-on machine learning with ScikitLearn, Keras, and TensorFlow: Concepts, tools, and techniques to build intelligent systems. O'Reilly Media.

78. Kingma DP, Welling M (2014) Auto-encoding variational bayes. In: 2nd Int Conf Learn Represent ICLR 2014 - Conf Track Proc International Conference on Learning Representations, ICLR.

79.Goodfellow IJ, Pouget-Abadie J, Mirza M, Xu B, Warde-Farley D, et al. (2014) Generative adversarial nets. arXiv:1406.2661v1.

80.Makhzani A, Shlens J, Jaitly N, Goodfellow I, Frey B (2015) Adversarial autoencoders. arXiv:1511.05644v2.

81. Yusuf SM, Gao N (2017) Influence of energy density on metallurgy and properties in metal additive manufacturing. Mater Sci Technol (United Kingdom) 33: 1269-1289.

82. Srivastava N, Hinton G, Krizhevsky A, Sutskever I, Salakhutdinov R (2014) Dropout: A simple way to prevent neural networks from overfitting. J Mach Learn Res 15: 1929-1958.

83.Ng AY (2004) Feature selection, L1 vs. L2 regularization, and rotational invariance. Proceedings, Twenty-First Int Conf Mach Learn ICML 2004, ACM Press, New York, USA, 615-622.

84. Koeppe A, Hernandez Padilla CA, Voshage M, Schleifenbaum JH, Markert B (2018) Efficient numerical modeling of 3D-printed lattice-cell structures using neural networks. Manuf Lett 15: 147-150. 